\title{
La imputación normativa en la responsabilidad civil extracontractual
}

The normative imputation in extra-contractual civil liability

Autor: Luis Daniel Trejos Teherán

DOl: https://doi.org/10.19053/16923936.v19.n37.2021.13004

Para citar este artículo:

Trejos Teherán, L. D. (2021). La imputación normativa en la responsabilidad civil extracontractual. Derecho y Realidad, 19 (37), 195-209. 


\section{LA IMPUTACIÓN NORMATIVA EN LA RESPONSABILIDAD CIVIL EXTRACONTRACTUAL*}

The normative imputation in extra-contractual civil liability

\section{Luis Daniel Trejos Teherán}

Abogado. Universidad Simón Bolívar, sede Cúcuta. Código ORCID: http://orcid.org/0000-0003-3088-2123

abg.danielteheran@gmail.com

Recepción: Septiembre 21 de 2020

Aceptación: Abril 6 de 2021

\section{RESUMEN}

En el presente artículo se abordan las diferentes teorías que se han creado sobre la causalidad aplicada en la responsabilidad civil, de allí que esta investigación tenga como objetivo desarrollar la imputación normativa como eje constitutivo de la responsabilidad civil, para lo cual se debe crear un test de aplicabilidad normativa, ya que -como se ha podido observar en la praxis judicial-el nexo de causalidad carece de algún tipo de test que le permita a los jueces aplicar de manera efectiva la causalidad. Así, mediante la construcción de matrices de análisis documental y la utilización de categorías emergentes desde la teoría fundamentada, se obtuvo que la teoría causalista posee falencias estructurales y que hacen necesario plantear un nuevo elemento estructurador que contenga un test de aplicabilidad que les otorgue a los operadores jurídicos las herramientas necesarias para endilgar la responsabilidad

* Artículo de reflexión civil de manera eficaz. También se obtuvo la creación y desarrollo de un test normativo de carácter científico y jurídico lo suficientemente fuerte para darle la solides respectiva al nuevo elemento estructurador como lo es la imputación normativa.

\section{PALABRAS CLAVES}

Daño; test; causalidad; responsabilidad civil; imputación objetiva.

\section{ABSTRACT}

This article deals with the different theories that have been created on causality applied in civil liability, hence the objective of this research is to develop normative imputation as a constitutive axis of civil liability, for which a test of normative applicability must be created, since -as has 
been observed in judicial praxis- the causal nexus lacks some kind of test that allows judges to effectively apply causality. Thus, through the construction of documentary analysis matrices and the use of emerging categories from grounded theory, it was obtained that the causalist theory has structural shortcomings and that it is necessary to propose a new structuring element that contains an applicability test that provides legal operators with the necessary tools to effectively attribute civil liability. The creation and development of a normative test of a scientific and legal nature strong enough to give the respective solidity to the new structuring element such as normative imputation was also obtained.

\section{KEYWORDS}

Damage; test; causation; civil liability; objective imputation.

\section{INTRODUCCIÓN}

El nexo causal como requisito de la responsabilidad civil, ha suscitado gran controversia ya que como lo sostiene Olivares et al (2018), la causalidad no es un elemento suficiente sino necesario para la atribución del deber de indemnizar un daño, que junto con el elemento daño y el elemento imputación- forman dicha atribución. Siguiendo esta línea argumentativa, Velásquez (2013) menciona que la causa del daño se puede atribuir a la conducta de (i) una persona y también a una (ii) causa externa o de la naturaleza. No obstante, en lo que concerniente a estas últimas, las mismas surgen como rompimiento del nexo de causalidad toda vez que dentro de los juicios de responsabilidad no existen causales exonerativas sino causales de rompimiento causal.

De lo anterior se puede entender que la causalidad ha sido insuficiente para la imputación del daño debido a los problemas estructurales que trae consigo. Con el ánimo de entender la teoría causalista a profundidad, se hace necesario realizar una distinción bastante precisa sobre lo que se debe comprender por causalidad jurídica y causalidad física. Puede existir causalidad física, mas no jurídica, es decir, desde el plano ontológico de la causalidad la misma nace de un plano natural que no tiene incidencia en el campo del derecho, ello es así porque su característica deviene en que su manifestación no procede de ninguna acción donde intervenga el ser humano, por ello, la causalidad jurídica está conformada por aquellas acciones humanas que tienen relevancia jurídica en tanto se lesionan o entran en detrimento aquellos derechos intersubjetivos de los individuos (Tamayo, 2008). De esta forma, se entiende de manera más clara la distinción que se debe hacer entre la causalidad física y la jurídica. Tal distinción es imperiosa, ya que -no en pocas ocasiones- se pueden llegar a confundir.

Ahora bien, es menester tener presente que hay, a su vez, dos tipos de causalidades; a saber, la (i) causalidad simple y la (ii) causalidad compleja. "Por causalidad simple se puede entender como aquella en la cual la víctima puede establecer un nexo causal entre el comportamiento del agente y el daño sufrido y por (ii) por causalidad compleja, se entiende como aquel cumulo de nexos causales que comprenden las causas y los efectos comportamentales de dicho agente respecto del daño ocasionado" (Tamayo, 2008, p. 251). De esta forma, se puede avizorar nuevamente el gran problema que trae consigo la teoría de la causalista, pues establecer el nexo entre una o más causas supone una complejidad de gran magnitud, debido al complejo diseño estructural que posee esta teoría haciendo que sea imposible demostrar probatoriamente la existencia del nexo causal.

Ahora, frente a los criterios de imputación la Corte Suprema de Justicia sostiene en sentencia SC-13925 de 2016 con reiteración imputacional en sentencia SC-002 de 2018- y, sumado a ello una gran gama de doctrinantes. Es bien sabido que nuestra jurisprudencia explicó -desde la primera mitad del siglo anterior- que el artículo 2356 del Código Civil consagra una presunción de culpa, de allí que solo sea necesario acreditar el daño y el nexo causal dentro del proceso de responsabilidad toda vez que para la imputación se debe 
observar el régimen subjetivo que se impone en la normatividad colombiana. Debido a lo expuesto anteriormente, surge como interrogante: ¿Cuál es la estructura de la imputación normativa como criterio de imputación de responsabilidad civil?

Siguiendo esta línea argumentativa, el nexo causal no en muy pocas ocasiones se torna complejo y problemático (Laborda, sf). Ello es así ya que la construcción del mismo se basa en hechos externos al mundo jurídico, es decir, a situaciones naturales que hacen complejas la ideación y la construcción de los enunciados jurídicos que toman forma jurídica.

Aunado a lo anterior, resulta necesario precisar que dentro de la imputación que se realiza mediante el nexo causal no existe un test de aplicabilidad del mismo, lo que hace casi imposible concebir cómo el juzgador puede realizar una adecuación rigurosa de las causas que pudieron originar el daño.

Por ello, la presente investigación es necesaria en la medida en que la observancia de nuevos elementos estructurales de la responsabilidad civil dentro del ordenamiento jurídico colombiano permite que haya una construcción teórica diversa, toda vez que la hegemonía que se ha realizado respecto del nexo causal como único criterio correlacional entre la conducta de un agente dañador y el resultado dañoso de esa conducta, ha conllevado a que en muchas ocasiones las demandas de responsabilidad sean denegadas toda vez que se hace imposible probar la relación causal explicativa entre el daño y el hecho dañoso.

Debido a la insuficiencia que supone la teoría causalista la cual ha sido imperante en el ordenamiento jurídico colombiano para la imputación del daño, se han adoptado a lo largo del tiempo algunas teorías de causalidad que intentan suplir unas y otras, los fallos estructurales de estas, ahora, como se verá más adelante, se realizara un análisis crítico de las teorías imputacionales que han sido aplicadas en el sistema jurídico colombiano.
Sin embargo, hay quienes sostienen que para la existencia de la responsabilidad civil se debe acreditar el nexo causal entendido este como aquella relación entre la conducta humana dañosa y el daño producido como resultado de esa conducta (Quiñonez \& Restrepo, 2014).

Debido a este elemento tiene una vasta tradición en la responsabilidad civil y Colombia no se ha quedado atrás. Ya que el artículo 2341 del Código Civil indica: "el que ha cometido un delito o culpa, que ha inferido daño a otro, es obligado a la indemnización (...)" (Código Civil, 1873) pero bajo este precepto se debe acreditar la conexión fáctica entre el daño y el hecho producido por el agente dañador, ya que sin este requisito no sería aplicable dicho artículo.

Y es que, es innegable que el nexo causal que se ha exigido para la imputación de la responsabilidad civil en el ordenamiento jurídico colombiano no ha sido lo suficientemente contundente $\mathrm{y}$, por lo tanto, se ha evidenciado con el pasar del tiempo que dicho nexo causal debe ser replanteado en su estructura.

De esta forma, Dosso sostiene que:

En lo fundamental distingue entre imputación al hecho e imputación a la culpabilidad; la primera -la imputación objetiva- no es otra cosa que la adecuación: "En vez de causalidad "adecuada", la ciencia del Derecho debería exigir causalidad "objetivamente imputable". $\mathrm{Si}$ el resultado no fue objetivamente previsible, el comportamiento de la persona que lo haya causado no debe ser considerado como su propio hecho, sino como un acontecimiento casual (Dosso Dal, 2011, p. 5).

La Corte Suprema de Justicia (2016) es contundente al señalar en la sentencia SC13925, que con cierta frecuencia se nieguen demandas de responsabilidad civil por no acreditarse en el proceso un "nexo causal" que es difícil de demostrar, porque existe como hecho de la naturaleza; es 
decir, imputar un daño por el criterio causal va encaminado a determinar si el hecho generador del daño es consecuencia natural, pues en caso de ser lo último se tendría que analizar que la teoría causalista es la aplicable al caso concreto, como quiera que cada teoría de estas solo suple falencias de antecedente.

Es de recordar que en la producción del daño confluyen muchas causas, las cuales hacen que sea prácticamente imposible de determinar cuál fue la originadora del daño, ya que sean cercanas o remotas, directas o indirectas, todas han influido en el hecho dañoso, por lo tanto la elección de alguna de ellas resultará excluyente de las demás y esto generará la imposibilidad probatoria del nexo causal de las víctimas al momento de incoar el proceso de responsabilidad civil; es por esto que a buena hora la Corte observó esta garrafal falencia en la estructura de la responsabilidad civil.

Por ejemplo, para establecer si una conducta (activa u omisiva) se puede atribuir a un agente hay que partir de categorías jurídicas como (i) el deber de actuar, (ii) las acciones y (iii) las omisiones relevantes, (iv) la posición de garante, (vi) el concepto de 'guardián de la cosa, (vii) las obligaciones de seguridad, etc., (que no llevan implícitos juicios de reproche), las cuales no se constatan directamente sino que se atribuyen a partir de un marco de sentido jurídico que permite la construcción de pruebas inferenciales.

Es por ello que se debe desprender la causalidad natural del plano de imputación jurídica de responsabilidad, toda vez que esta no constituye el proceso de atribución de un hecho jurídico a un agente, porque la cualidad de artífice se encuentra prefigurada por una concepción normativa, o sea que cada comportamiento es valorado dentro de un horizonte de conductas que se erige como patrón selectivamente relevante.

Existe una diferencia fundamental entre el principio de causalidad y el de razón suficiente, toda vez que el primero busca el origen material de un hecho que se encuentra mediante la selección de relaciones causales, en tanto que el segundo se pregunta por qué un resultado puede ser atribuido a una acción dentro de un marco de valores preestablecidos desde la explicación de las razones que anteceden a la conducta.

Una persona puede originar un hecho desencadenante de un daño y, sin embargo, el nexo causal por sí solo resulta irrelevante para endilgarle ese hecho como suyo; como bien puede ocurrir que la autoría del hecho lesivo deba ser asumida por quien no tuvo ninguna intervención o injerencia física en el flujo de eventos que ocasionaron el daño.

Así, Olivares et al (2018) menciona que para la existencia de una relación de causalidad, se hace necesario que un sujeto haya determinado el resultado con una acción proporcionadora, adecuada. Sin embargo, desechar alguna de las causas que pudieron confluir en la producción del daño haría que tanto las que desechan como la que se utiliza, lleven a la errónea apreciación de la prueba causal dentro del proceso de responsabilidad civil.

Esto por cuanto la valoración de la probatoria para la demostración del nexo causal debe partir desde el plano causal natural para lograr la adecuación jurídica de las implicaciones que conllevan las causas naturales dentro del plano jurídico y es que dicho nexo no se prueba directamente, sino que se plantean hipótesis inferenciales con base en probabilidades (Corte Suprema de Justicia, 2016). Es importante mencionar que dichas hipótesis parten de un plano subjetivo que permean las probabilidades de demostrar el nexo causal, ya que como se mencionó ut supra, el nexo causal existe como hecho de la naturaleza y la atribución de un hecho a un agente se realiza mediante las funciones sociales y de responsabilidad que le establece un ordenamiento jurídico.

De lo anterior se puede entender que la relación causal entre el agente de conducta dañosa y el hecho que genera el daño no siempre da una explicación del accionar (acción u omisión), esto debido a la insuficiencia explicativa que ofrece 
dicho nexo, puesto que adolece de fallas estructurales.

Se ha afirmado que tal presunción se desvirtúa con la demostración de una causa extraña a la conducta del agente, por lo que es intrascendente la prueba de la prudencia socialmente esperable. A partir de los riesgos del sistema de producción industrial y sus daños actuales o potenciales se da una pluralidad casi infinita de interpretaciones causales que no pueden ser confirmadas.

\section{METODOLOGÍA}

De esta manera, se fijó el paradigma interpretativo desde un enfoque cualitativo, ello permitió analizar a profundidad las diferentes teorías causalistas que se han elaborado, de esta manera desarrollar el test de aplicabilidad normativa (Martínez, 2009) y bajo el diseño de la teoría fundamentada se recurre como método al hermenéutico. Como fuentes de información se tomaron la jurisprudencia y los postulados teóricos, los cuales son abordados mediante la técnica de análisis documental desde el discurso, tomando como instrumento una matriz de análisis planteada por Hurtado (2010).

Así, para dar un orden lógico a la investigación y de acuerdo al alcance de la misma, se constituyen como objetivo general el desarrollar la imputación normativa como eje constitutivo de la responsabilidad civil y, como objetivos específicos (i) Crear un test de aplicabilidad que sustente la teoría de la imputación normativa, para seguidamente (ii) Diseñar un estándar probatorio para la imputación normativa (iii) Implementar un modelo practico de aplicación normativa de la imputación.

\section{RESULTADOS}

\section{Teorías sobre el nexo de causalidad}

Debido a la insuficiencia que supone la teoría causalista la cual ha sido imperante en el ordenamiento jurídico colombiano para la imputación de un daño y, por consiguiente, la generación de la responsabilidad civil, por ello se expondrán infra algunas de las teorías causalistas que han sido aplicadas en Colombia.

Tamayo (2008) expone alguna de las teorías causalista imperantes en la tradición jurídica colombiana, a saber: "1) la equivalencia de las condiciones, 2) la causalidad adecuada, 3) la causa próxima, 4) la causa eficiente, 5) la de la acción humana" (Tamayo, 2008, p. 375) entre otras, pero con el objeto de identificar las más utilizadas por la doctrina y la jurisprudencia colombiana se analizarán las dos primeras.

\section{TEORÍA DE LA EQUIVALENCIA DE LAS CONDICIONES}

Dalcq, citado por Tamayo (2008), sostiene que: "el punto fundamental de esta teoría es la idea de que cada uno de los eventos que son la condición de la realización del daño juega en esta realización un papel causal idéntico" (Tamayo, 2008, p. 376), es decir, que todas las causas que confluyen en la producción del daño son todas del mismo rango y ninguna se puede excluir.

Consecuentemente, "la teoría de la equivalencia de las condiciones es la única doctrina que explica en forma científica el problema del nexo de causalidad" (Tamayo, 2008, p. 377), sin embargo, dicha teoría presupone la extensión de la causalidad ad infinitum, en otras palabras, que su aplicabilidad práctica en sí misma conllevaría a hacer responsable a toda persona que de una u otra manera pudiera haber intervenido en la producción del perjuicio.

El planteamiento arquitectónico de esta teoría es que no se puede realizar ninguna distinción causal explicativa de la producción de un daño (Guiñez, 2010), toda vez que el análisis por separado bajo esta teoría no tendría la suficiencia para el entendimiento respecto de la producción del daño.

Causalidad adecuada. Como se puede observar, que de la imposibilidad práctica que presupone la teoría de la equivalencia de las condiciones se diseñó -como enmienda estructural- la segunda teoría a estudiar y 
que actualmente es la utilizada en el sistema de responsabilidad colombiano, como lo es la causalidad adecuada al momento de imputar la responsabilidad civil.

A le da un golpe en la cabeza a B, insuficiente para provocar una lesión en un ser normal. Pero ocurre que B está afectado de una debilidad congénita de los huesos del cráneo y golpe leve basta para matarlo. La teoría de la causalidad adecuada conduciría a considerarla debilidad particular de los huesos del cráneo de B como un evento excepcional, $\mathrm{y}$ a considerar desde luego que el acto de A no es la causa del daño porque esta consecuencia no era previsible. Por el contrario, si se hubiera tratado de un niño, A habría podido prever semejante debilidad y su acto habría sido considerado como la causa adecuada de la muerte del niño (Tamayo, 2008, p. 379).

Del ejemplo anterior, se puede entender como la teoría de la causalidad adecuada se torna insuficiente al momento de establecer el nexo causal en la producción de un perjuicio, "pues como se observa los defensores de esta teoría asumen la indagación del nexo causal desde el terreno psicológico para dar una explicación práctica" (Tamayo, 2008, p. 379), esto obliga a entender que los antecedentes que pudieron originar el perjuicio son los que explican el nexo causal, así, pues, esta teoría pretende la eliminación de aquellos antecedentes, que no han ayudado en la producción del daño.

Ahora bien, como se ha expuesto anteriormente, para la existencia de la responsabilidad civil es necesario exista un nexo causal entre el hecho y el daño, siendo este último de gran relevancia para la endilgación de la responsabilidad.

Henao respecto al daño, afirma: "establece que es la razón de ser de la responsabilidad, y por ello, es básica la reflexión de que su determinación en sí, precisando sus diversos aspectos y su cuantía, ha de ocupar el primer lugar, en términos lógicos y cronológicos, en la labor de las partes y juez en el proceso" (Henao, 2007, p. 35).
Desde la postura inicial del autor se puede entender que el daño es un elemento indispensable para la existencia de la responsabilidad, pues si no hubo daño o es imposible probar la existencia del mismo, la imputación que se realizaría para la existencia de la responsabilidad sería vaga e inútil.

Como se mencionó, el daño constituye una regla esencial en la existencia de la responsabilidad, pero es menester advertir que toda regla tiene una excepción:

Sin embargo, en ocasiones a pesar de existir daño no procede declarar la responsabilidad. Esto por cuanto el daño es requisito indispensable más no suficiente para que se declare la responsabilidad. En efecto, en algunos eventos ocurre que no se declara la responsabilidad a pesar de existir un daño (Henao, 2007, p. 38).

Esto pone de presente que el daño es una condición necesaria más no suficiente para la existencia de la responsabilidad. La anterior afirmación conlleva a pensar que no siempre procede la declaratoria de responsabilidad aun cuando se puede comprobar la ocurrencia de un daño:

En efecto, en algunos eventos no se declara la responsabilidad, a pesar de haber existido el daño. Es lo que ocurre en dos hipótesis: el daño existe, pero no se puede atribuir al demandado, como cuando aparece demostrada una de las causales exonerativas; o el daño existe y es imputable, pero el imputado no tiene deber de repararlo, porque no es un daño antijurídico y debe ser soportado por quien lo sufre (Henao, 2007, p. 38).

\section{NUEVA ESTRUCTURA DE LA RESPONSABILIDAD CIVIL EXTRACONTRACTUAL}

\section{El Daño}

Respecto al daño, Henao establece: "que es la razón de ser de la responsabilidad, y por ello, es básica la reflexión de que su 
determinación en sí, precisando sus diversos aspectos y su cuantía, ha de ocupar el primer lugar, en términos lógicos y cronológicos, en la labor de las partes y juez en el proceso" (2007, p. 35).

Desde la postura inicial del autor se puede entender que el daño es un elemento indispensable para la existencia de la responsabilidad, pues si no hubo daño o es imposible probar su existencia, la imputación que se realizaría para existencia de la responsabilidad sería vaga e inútil.

Como se mencionó anteriormente el daño constituye una regla esencial en la existencia de la responsabilidad, pero es menester advertir que toda regla tiene una excepción, el daño es una condición necesaria más no suficiente (Henao, 2007) y el estudio del daño ha sido uno de los temas controversiales para la responsabilidad, puesto que ha de analizarse desde el caso concreto, por ello, ha de ser el daño el primer requisito a estudiar para entrar a determinar la existencia de un proceso de responsabilidad (Velásquez, 2013; Henao, 2007) de allí que se entienda su condición de necesidad mas no la de suficiencia, toda vez que sin la existencia de este se haría innecesario estudiar los demás elementos estructuradores.

Daño probado. Como se expuso anteriormente, el daño es el primer elemento a estudiar para que pueda existir la responsabilidad civil, no obstante, existe una característica especial para este primer elemento y es por ello que se ha exigido desde la jurisprudencia y la doctrina- que el daño sea probado.

Para la acreditación del daño existen unos medios probatorios que hacen imprescindible tal razón, de ahí que el artículo 167 del Código General del Proceso establece que la carga de la prueba a quien alega haber sufrido un daño cuando menciona que "incumbe a las partes probar el supuesto de hecho de las normas que consagran el efecto jurídico que ellas persiguen (...)" (Código General de Proceso, 2012) de ello se entiende que la parte que alega un daño se valga de todos los medios conducentes para probar la existencia de este elemento estructurador de ahí, pues, que surje el principio de carga de la prueba del daño, el cual tiene dos características:

Figura 1. Existencia y cuantificación del daño

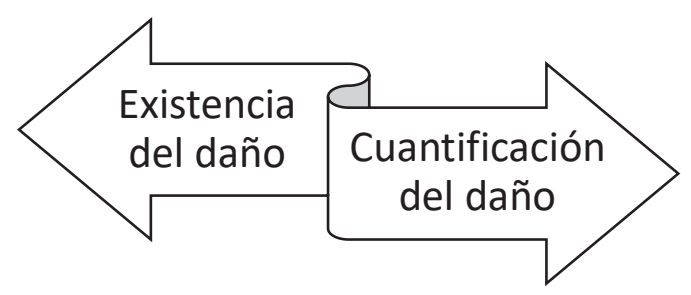

Fuente: Informe de Inspección

Existencia del daño. Alterar la existencia de un estado de cosas que se pueda probar por la parte del proceso que la alega, es decir, el daño debe ser real y debe existir una certeza de la existencia del mismo, ello será posible mediante los diversos medios probatorios existentes en el ordenamiento jurídico colombiano.

Cuantificación del daño. Esta característica es imprescindible ya que permite establecer un estándar mínimo para su cuantificación, de ahí que surjan las siguientes reglas:

- Regla 1. Indemnización total del daño. La esencia de la responsabilidad civil es ser resarcitoria, es decir, colocar o dejar en el estado en que se encontraba una persona o los bienes de esta afectados antes de la ocurrencia de un hecho dañoso, eso quiere decir que la indemnización debe estar en una medida equitativa para las partes, es decir, no puede estar por encima de lo que se estima ha sido el desmejoramiento de un derecho patrimonial o extrapatrimonial, ya que se generaría un enriquecimiento sin causa para la parte que recibe el resarcimiento, ni tampoco debe estar por debajo de lo equitativo porque originaría empobrecimiento sin causa puesto que desmejoraría las pretensiones de 
la parte actora dentro del proceso de responsabilidad civil. - Regla 2. El proceso no determina
la indemnización. La idea de esta regla es muy práctica, ya que dentro de ordenamiento jurídico colombiano existe una gran variedad de procesos que pretenden una resarcir la causación de un daño. A manera de ejemplo se puede encontrar en el Código de Procedimiento Administrativo y de lo Contencioso Administrativo una serie de medios de control que están encaminados a resarcir un daño como lo es el Control Inmediato de Legalidad (Art.36), Nulidad (Art.37), Nulidad y Restablecimiento del Derecho (Art.138), Nulidad Electoral (Art.139), Reparación Directa (Art.140), Controversias Contractuales (Art.141), Repetición (Art.142),Perdida de Investidura (Art.143), Protección de los Derechos e Intereses Colectivos (Art.144), Reparación de los Perjuicios Causados a un grupo (Art.145), Cumplimiento de las Normas con Fuerza Material de Ley o Actos Administrativos (Art.146) Nulidad de las Cartas de Naturaleza (Art.147) y Control por Vía de Excepción (art.148).

Ya en el área civil es un poco más sencillo, esto debido a que existe un proceso de Responsabilidad Civil Contractual o Extracontractual dependiendo del caso concreto. Este tipo de procesos se pueden encontrar en el libro tercero, sección primera, título I, capítulo I del Código General del Proceso, relativo a los procesos verbales; sin embargo, comparte una misma raíz con los diversos medios de control enmarcados en el Código de Procedimiento Administrativo y de lo Contencioso Administrativo, esto es, buscar un resarcimiento de un daño irrogado por una persona jurídica o natural.

\section{EL HECHO}

Estudiar este elemento estructurador de la responsabilidad civil se torna complejo en la medida que no existe una definición o tan siquiera un concepto al menos vago o difuso del mismo, por ello, se hace imprescindible conocer que es un hecho desde una aproximación teórica, para poder dar una conceptualización respecto de este elemento.

Y es por ello que -para poder entender que es un hecho dentro de la responsabilidad civil-se debe remitir a un concepto científico del mismo. Existen cinco características del hecho científico: (i) el hecho está a merced del investigador, es decir, es la premisa constitutiva de una teoría, como quiera que la utilización del mismo dentro del proceso tendrá que estar sujeto a los medios probatorios de los que disponga la parte que alega la ocurrencia del mismo, sin embargo, estos (ii) hechos necesariamente deben estar por fuera de la teoría debido a que debe existir una correspondencia pero a la vez una separación entre los hechos y la teoría para que pueda ser lo suficientemente sólida y permanecer en el tiempo, se comprende entonces que la correspondencia está dada en la medida en que la relación entre los hechos y la teoría es lo suficientemente fuerte aun cuando el análisis probatorio que se realice sea tan exigente que así no se pueda desligar el uno del otro, (iii) los hechos deban ser auténticos a fin de que puedan confirmar o refutar una teoría y es que la correspondencia entre ambos elementos le otorga el criterio de admisibilidad a la teoría pero, tal admisibilidad está constituida por la (iv) falta de variación de los hechos, es decir, debido a que los hechos están en el exterior de la teoría la invariabilidad permite que su autenticidad soporten la refutación de una determinada teoría y finalmente (v) los hechos aunque deben estar por fuera de la teoría no deben estar totalmente desligados ya que estos entonces no podrán corregir su construcción teórica y no servirían como criterios de autenticidad para la teoría (Díaz, Calzadilla y López, 2004).

Ahora bien, de lo reseñado anteriormente se puede entender que un hecho es un acontecimiento que modifica el estado de las cosas en general, que puede ser humano o natural, dependiendo de su forma de manifestarse y podrá tener relevancia jurídica, es por eso que se puede considerar como la premisa menor dentro del supuesto de la Responsabilidad Civil; esto es, que 
dependerá del hecho jurídicamente relevante para la existencia de la responsabilidad civil, en la medida que exista coherencia y correspondencia con el supuesto daño que alega una parte dentro del proceso.

Cabe resaltar que dentro del derecho de la responsabilidad no existen eximentes de la misma, sino formas de romper el nexo causal como lo es la (I) culpa exclusiva de la víctima, (II) la culpa de un tercero y/o (III) un caso fortuito. Vale aclarar este punto, ya que ha habido cierta confusión respecto de si esos tres componentes exoneran de responsabilidad a un supuesto agente dañador $y$, es de precisar que -aunque el daño sea cierto y haya correspondencia entre este y el hecho- se debe entender que el rompimiento de dicho nexo causal ase presenta debido a un elemento ajeno a la voluntad del agente dañado supuesto.

\section{IMPUTACIÓN NORMATIVA}

Como ya se mencionó, la responsabilidad civil ha tenido una estructura clásica de antaño consistente en el Daño + Hecho + Nexo causal. Esta trilogía jurídica ha tenido fallas estructurales por lo que resulta pertinente una exploración analítica en el sistema jurídico colombiano, con el objetivo de encontrar un nuevo elemento que pueda ser utilizado en la arquitectura de la responsabilidad civil y que sea más confiable al momento de su utilización respecto del juicio de responsabilidad. Dicha trilogía había sido intocable en el ordenamiento jurídico colombiano y, a su vez, pertinente en la resolución de problemas judiciales respecto de los procesos de responsabilidad civil, según se ha dejado entrever como uno de sus elementos estructurales.

Desde la doctrina nacional e internacional y desde la jurisprudencia emanada por la Corte Suprema de Justicia se han estudiado a profundidad de los elementos estructurales de la responsabilidad en donde se advierten los yerros que trae consigo la causalidad y en específico el nexo causal, ya que este es uno de los muchos elementos de los que se valen los ordenamientos jurídicos para la imputación del daño. Este criterio no solo era necesario para endilgación de la responsabilidad, sino que envuelve un sinfín de problemas probatorios, ya que dependiendo de la teoría causalista que se escogiera para generar de esta manera la imputación y la consecuente responsabilidad, en muchas ocasiones se tornaba difícil casi que imposible dicho nexo, de ahí que con gran frecuencia se denieguen demandas de responsabilidad.

No solo debe advertir que el nexo causal es un elemento de los muchos que existen en el ordenamiento jurídico colombiano, sino que para realizar una reestructuración de la responsabilidad civil se debe observar dentro del mismo para poder encontrar un nuevo criterio que supere las falencias estructurales de la causalidad. Es por ello que surge la imputación normativa, es decir, un elemento normativo puro (Ver Ilustración 2).

Figura 2. Nueva Estructura de la Responsabilidad Civil
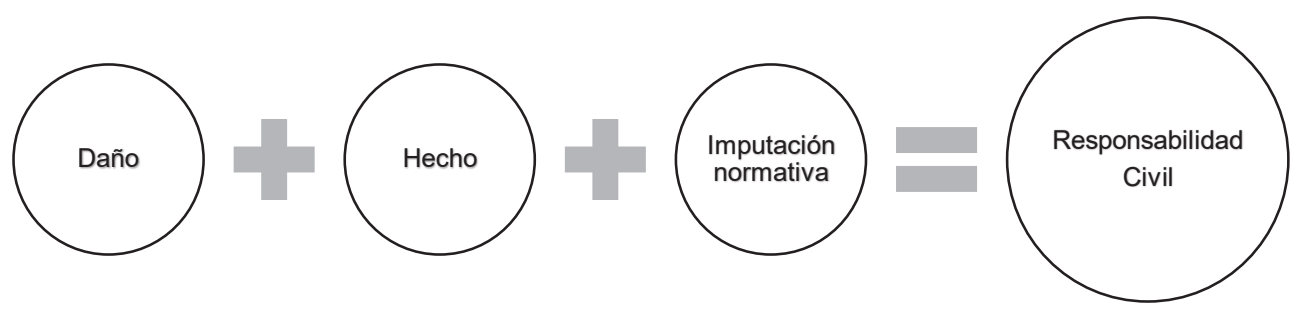

Fuente: Elaboración propia. 
Esta nueva estructura constituye un cambio paradigmático, ya que este nuevo elemento modifica uno que había sido considerado como esencial para la endilgación de la responsabilidad; de esta manera este avance da cuenta de los muchos criterios que tienen los ordenamientos jurídicos y en especial el ordenamiento jurídico colombiano para realizar la imputación del daño.

\section{PRECISIONES SOBRE LA IMPUTACIÓN NORMATIVA}

Con la imputación normativa se puede observar cómo dentro del ordenamiento jurídico colombiano existen otros criterios para formular la responsabilidad civil, que mediante el análisis introspectivo de los sistemas objetivo y subjetivo de imputación se da nacimiento a este nuevo elemento que sin duda es de gran relevancia teóricopráctica, pues no es fácil -por ejemplo dentro de la academia- concebir la responsabilidad civil sin la causalidad; empero, a partir de esta investigación se debe comenzar a buscar otros elementos normativos que sirvan como base de imputación dentro del área jurídica.

Observar qué norma jurídica se ajusta al caso concreto, teniendo como base la conducta del agente y el resultado de su actuar (hecho dañoso) es lo que estructura la atribución de la responsabilidad civil desde la imputación normativa. Nótese cómo se pueden abordar nuevos elementos desde la academia que permiten una ampliación y una reestructuración de la responsabilidad civil. De acuerdo con lo anterior, es innegable que este cambio genera un choque cognoscitivo en la forma como se ha concebido la estructura clásica la responsabilidad, sin embargo, el mismo es más que necesario para que se presente una actualización en la manera como se ha estructurado desde hace mucho tiempo esta área del derecho en nuestro ordenamiento jurídico.

De lo anterior nace el cambio de criterio, ya que -como se dijo anteriormente- la prueba del nexo causal se debe remitir a hechos de la naturaleza, lo que genera la imposibilidad probatoria del mismo. Si, por el contrario, la imputación obedece a criterios normativos, la conducta dañosa debe encarrilar el proceso de responsabilidad civil y adecuar la conducta del agente dañado con el elemento fáctico-normativo.

Es de allí de donde radica el cambio paradigmático, puesto que no solo se está reestructurando la responsabilidad, sino que se comienza a volcar la mirada en una cuestión que era casi imposible en el derecho de la responsabilidad, ya que al ser una tradición la imputación mediante la causalidad, la falta de ampliación visual normativa permitía concebir que el nexo causal era el único requisito existente para la imputación del daño.

En este punto es necesario diferenciar la imputación civil de la imputación penal, ya que la última lo que busca es un responsable de punible y su pena y/o sanción, lo cual es completamente distinto de la imputación de la responsabilidad civil, ya que esta lo busca es el resarcimiento del daño; es decir, es una imputación resarcitoria.

\section{DIFERENCIAS ENTRE LA IMPUTACIÓN NORMATIVA E IMPUTACIÓN OBJETIVA}

Siguiendo con la línea argumentativa, el cambio de criterio de imputación, esto es la imputación normativa, se podría llegar a confundir con la imputación objetiva del derecho penal; sin embargo, la diferenciación radica en que el derecho penal busca la adecuación típica de la conducta del infractor de la ley penal para que consecuentemente responda por su actuar, por medio de la imposición de una pena lo que no sucede en con la imputación normativa, ya que no se busca la imposición de una pena sino el resarcimiento del daño causado ya sea patrimonial o extramatrimonial.

La prueba de la imputación del hecho a un agente no se puede establecer únicamente a partir del análisis de la "causalidad natural pura" o, en otras palabras, solo por el nexo causal, porque las explicaciones físicas o mecánicas del comportamiento generador de un resultado no siempre son 
distinciones indiscutibles en el lenguaje jurídico y nunca lo son en materia de omisiones y responsabilidad indirecta, ya que la imputación deviene de una situación que no se agotan en los hechos generadores del daño, sino que tales hechos cobran relevancia al hacer dicha imputación, de esta manera la labor que ejerce el imputante es la de analizar dentro del universo de causas, las cuales fueron las que confluyeron para la realización del daño; es decir, que las preconcepciones jurídicas de este son las que valoran la relevancia y posterior selección de los hecho (s) dañoso (s), que se ajustan al sistema normativo a fin de que se le pueda endilgar la responsabilidad al presunto agente dañador.

Así encuentra fundamento la imputación normativa, ya que si bien es cierto en muchos casos las conductas de agentes dañadores no tiene una positivización, también es cierto que el nexo causal no explica la relación entre su actuar y el daño que percibe una persona (s), por lo tanto se hace necesario dar una mirada más allá de la causalidad, puesto que como ha quedado demostrado esta tiene una gran falla estructural y se hace imprescindible recabar dentro del ordenamiento jurídico otros parámetros de imputación del daño para la endilgación de la responsabilidad. De esta manera, la imputación normativa se constituye en un criterio existente en el ordenamiento jurídico colombiano para dicha imputación.

$Y$ es que en efecto, aunque muchas conductas no se encuentren en un inicio proscritas como generadoras de la responsabilidad civil, un análisis a profundidad del contenido normativo del ordenamiento jurídico dará cuenta de que existe un catálogo amplio de normas que traen consigo el establecimiento de una conducta del agente dañador como guardián de tal accionar y esta será la norma que servirá al juzgador para endilgación de la misma como obra del tal agente dañador, de suerte que el hecho presumible es la posibilidad de imputar el daño al demandado y, una vez demostrada esta imputación, habrá que dar por probada la culpa que menciona ese enunciado normativo, pues al no requerir demostración es un hecho presunto, lo anterior no es más que la endilgación de la responsabilidad civil mediante la imputación normativa.

\section{EL TEST DE APLICABILIDAD NORMATIVA PARA LA IMPUTACIÓN DEL DAÑO}

El nexo causal no posee un test que permita inferir la manera como el juzgador realiza la adecuación entre las causas que originaron el daño y los hechos que llevaron al mismo, por ello se hace necesario desarrollar un test de aplicabilidad normativa. Y es que, mediante el método deductivo, se puede llegar a una concreción respecto de la imputación del daño, de ahí que sea imperativa la creación y el desarrollo de un test que tenga una funcionabilidad formal para la endilgación del daño para el surgimiento de la responsabilidad civil, de ahí que se establezcan los siguientes pasos:

\section{Ilustración 3. Test de aplicabilidad normativa}

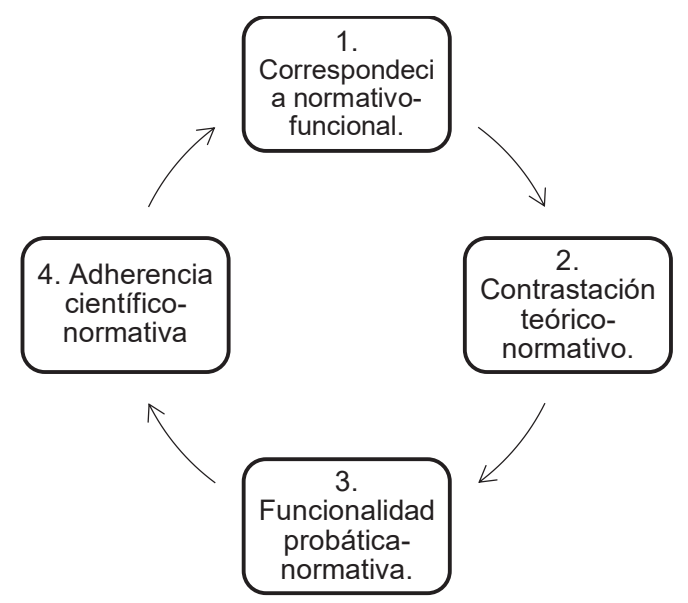

Fuente: Elaboración propia.

1. Correspondencia normativofuncional. Para la existencia de una correspondencia entre la norma y su función, se debe observar dentro del ordenamiento jurídico la norma rectora de la responsabilidad civil 
extracontractual. En el artículo 2341 del código civil colombiano establece que: "el que ha cometido un delito o culpa, que ha inferido daño a otro, es obligado a la indemnización, sin perjuicio de la pena principal que la ley imponga por la culpa o el delito cometido", no obstante, se debe analizar cuál es la funcionalidad de la misma.

El precitado artículo indica que quien infiera daño a otro es obligado a indemnizar, se encuentra explícitamente condensada la particularidad normativa de imputación y ello quiere decir que se necesita auscultar la existencia de una relación causal para realizar la imputación, toda vez que esta obedecerá a patrones normativos puros, condensados precisamente en la normatividad regulatoria de la responsabilidad civil extracontractual.

Es por ello que la correspondencia normativo-funcional está dada en la manera que el imputante del daño no solo observa los hechos y los (el) daño (s), sino que también analiza la norma y su función de manera que la generación de la imputación se desprenda de todo elemento natural.

Respecto de la correspondencia normativo-funcional, el análisis de la norma aplicable al caso concreto, estará orientada desde el estudio del daño y de los hechos generadores del daño, ya que esto permitirá descubrir la función de la norma en el caso sub examine.

\section{Contrastación teórico-normativo.}

Esta contrastación está cimentada en la idea de darle cientificidad a la imputación: es decir, es buscar entre la norma jurídica y la doctrina, una armonía normativa que genere una imputación no solo aislada de todo elemento natural, sino que también vaya en consonancia con lo establecido en el código civil.

La línea argumentativa construida desde la contrastación teórico-normativa, permitiría darle mayor cientificidad a la imputación, ya que los datos recaudados para esta última deberán estar orientados en virtud de la normativa jurídica que regule la responsabilidad civil.

No obstante, se puede presentar una dicotomía entre la teoría y la norma por ello, se hace necesario que la labor de operador judicial este orientada en función de la norma jurídica y no en elementos de índole natural que conlleven a la utilización de teorías cuasi-jurídicas que tornen ineficaz el juicio de imputación del daño.

\section{Funcionalidad probática-} normativa. La funcionalidad está orientada a la forma como se utilizan los diversos medios de prueba existentes en el ordenamiento jurídico, de ahí que sea tan importante la manera como estos están en función de la demostración de los elementos fácticos que acontecen dentro de la responsabilidad civil.

Lo anterior permite colegir que cada hecho jurídico lleva de manera subyacente una prueba para su demostración y que los primeros deben estar orientados en probar que la norma que supone la existencia de la responsabilidad civil pretendida se ha cumplido a cabalidad, ya que de lo contrario se tornaría ineficaz al momento de iniciar con el trámite de dicho proceso.

\section{Adherencia científico-normativa.} Esta adherencia tiene una íntima relación con la funcionalidad probáticanormativa, ya que los medios probatorios que se utilicen para la existencia de la responsabilidad civil deben estar acorde a las reglas científicas que se han diseñado y que son aplicables a cada medio probatorio.

Quiere decir lo anterior, que para tal demostración de estos elementos normativos en un caso concreto es fundamental el apoyo de la ciencia por medio de los diversos elementos científicos que estén encaminados a tal 
demostración. No solo es la utilización de teorías científicas que hayan superado la fase del debate teórico-práctico, sino que además se debe demostrar como dicha teoría es aplicable para la solución del caso que se estudia.

\section{CONCLUSIONES}

La causalidad no es el único elemento que existe en los ordenamientos jurídicos utilizables para la endilgación de la responsabilidad civil y, debido a que este tiene falencias estructurales se han creado diversas teorías que tratan de suplicar los diferentes fallos estructurales, por ello se hace necesario observar con mayor detenimiento los elementos imputacionales existentes en cada sistema jurídico y así, después de un análisis sistémico de las normas que permitan la endilgación de la responsabilidad civil, se pueda encontrar que el elemento de la imputación normativa se puede convertir en un nuevo criterio estructurador de la responsabilidad civil.

Ahora bien, con la creación y el desarrollo del anterior test se le otorga a la imputación normativa la solidez suficiente para remplazar la teoría causalista como criterio constitutivo de la responsabilidad civil; de esta manera, se dan alternativas que pueden cambiar la hegemonía tradicional en la estructura de la responsabilidad civil que había sido consistente en el Daño + Hecho + Nexo causal. Este es un test práctico que ayuda a los operadores jurídicos para realizar la endilgación de la responsabilidad civil de manera más ordenada y coherente con los postulados que se han erigido en el campo del derecho de daños.

La causalidad ha servido a la endilgación de la responsabilidad civil, a la doctrina y a la jurisprudencia del tema. Se han avizorado falencias, sin embargo, debido a que no existe una teoría que sea lo suficientemente estable y práctica para su reemplazo aún se sigue utilizando en los juicios de responsabilidad.

Surge como teoría restructuradora la imputación normativa, la cual fue utilizada por la Corte Suprema de Justicia en un par de sentencias, pero tal como fue manejada en dichos fallos no serviría sino como referente para futuros cambios teóricos del sistema de responsabilidad civil, es por ello, que la conceptualización teórica que aquí se realiza le dará cuerpo a esta teoría y -con la creación de test- se obtendrá solidez respecto de su aplicación y funcionalidad dentro de los procesos de responsabilidad.

\section{REFERENCIAS BIBLIOGRÁFICAS}

» Diario Oficial No. 47.956 (2011). Ley 1437. Por la cual se expide el Código de Procedimiento Administrativo y de lo Contencioso Administrativo.

» Diario Oficial No. 2.867. (1873). Ley 84. Por medio de la cual se expide el Código Civil De Los Estados Unidos De Colombia.

» Diario Oficial No. 48.489. (2012). Ley 1564. Por medio de la cual se expide el Código General del Proceso y se dictan otras disposiciones.

» Díaz V., Calzadilla A. \& López H. (2004). Una Aproximación al Concepto de Hecho Científico. Revista austral de ciencias sociales. http://mingaonline.uach.cl/ pdf/racs/n8/art01.pdf

» Aramburo, M. (2008). Responsabilidad civil y riesgo en Colombia: apuntes para el desarrollo de la teoría del riesgo en el siglo XXI. Revista facultad de derecho y ciencias políticas. Vol. 38, núm. 108. Recuperado de: https://www.redalyc.org/ pdf/1514/151413541002.pdf

» Colombia. Corte Suprema de Justicia (2016). Sala de Casación Civil (treinta de 
septiembre de dos mil dieciséis). Sentencia SC-139252016. (M.P. Ariel Salazar Ramírez).

» Colombia. Corte Suprema de Justicia (2018). Sala de Casación Civil (doce de enero de dos mil dieciocho). Sentencia SC-0022018. (M.P. Ariel Salazar Ramírez).

» Cordech, P. \& Crende, A. (2006). Causalidad y responsabilidad. Revista para el análisis del derecho. Recuperado de: http://www.indret.com/pdf/329_es.pdf

» Dosso Dal, D. (2011). La teoría de la imputación objetiva (tesis de maestría, Universidad de Mendoza). Recuperado de: http://master.us.es/cuadernosmaster/8. pdf

» Guiñez, R. F. (2010). Las teorías tradicionales sobra la causalidad. Revista de derecho y ciencias penales: Ciencias Sociales y Políticas, (14), 23-32. Recuperado de: file:///D:/Downloads/Dialnet-LasTeoriasTradicionalesSobraLaCausalidad-3337331.pdf

» Henao, J. (2007). El daño: análisis comparativo de la responsabilidad extracontractual del Estado en derecho colombiano y francés. Universidad Externado de Colombia.

» Laborda E. (sf). La valoración del daño: estudio del nexo de causalidad. http://www.aeds.org/congreso/congresos-aeds/Comunicaciones\%20Libres/ Nueva\%20carpeta/Eugenio\%20Laborda\%20.pdf

» Martínez Miguelez, M. (2009). Ciencia y arte de la metodología cualitativa. México. Trillas

» Olivares-Torres, F.Y., Aguilar-Barreto, A.J., Trejos-Teherán, L.D., y Gómez-Rodríguez, D.L. (2018).La imputación normativa en la responsabilidad civil: estudio de caso Guillermo Pulgarín Sossa y otros contra inversiones médicas y la clínica Las Vegas Coomeva. En Aguilar-Barreto, A.J., Hernández-Peña, Y.K., Contreras-Santander, Y.L., Flórez-Romero, M. (Eds.), La Investigación Sociojurídica: Un análisis de la incidencia de los aspectos sociales para el derecho (pp. 38-51). Cúcuta, Colombia: Ediciones Universidad Simón Bolívar.

» Pevrot, J. (2010). El problema de la relación de causalidad en el derecho de la responsabilidad civil. Revista Chilena de Derecho Privado. № 15, pp. 143-178

» Quiñonez, S. \& Restrepo, J. (2014). De la causalidad adecuada a la imputación objetiva en la responsabilidad civil colombiana. 129 Vniversitas, pp. 187-235. Recuperado de: http://dx.doi.org/10.11144/Javeriana.VJ129 doi:10.11144/Javeriana. VJ129.caio

» Roca, E. (2009). El riesgo como criterio de imputación subjetiva del daño en la jurisprudencia del Tribunal Supremo español. Recuperado de: http://www.indret. com/pdf/688_es.pdf

» Tamayo, J. (2008). Tratado de responsabilidad civil. t.I. Legis.

» Velázquez, O. (2013). Responsabilidad Civil Extracontractual. Segunda edición. Temis. 